\title{
Cardiovascular Disease-related Health Beliefs and Lifestyle Issues Among Karen Refugees Resettled in the United States From the Thai-Myanmar (Burma) Border
}

\author{
Akiko Kamimura' ${ }^{1}$ Kai $\mathrm{Sin}^{2}$, Mu Pye${ }^{2}$, Hsien-Wen Meng ${ }^{2}$ \\ ${ }^{1}$ Department of Sociology, University of Utah, Salt Lake City, UT; ${ }^{2}$ Department of Health, Kinesiology and Recreation, University of Utah, Salt Lake \\ City, UT, USA
}

Objectives: Refugees resettled in the US may be at risk for cardiovascular disease (CVD). However, little is known about CVD-related issues among Karen refugees who have migrated to the US from the Thai-Myanmar border. The purpose of this study was to examine CVD-related health beliefs and lifestyle issues among Karen refugees resettled in the US.

Methods: Karen refugees resettled in the US from the Thai-Myanmar border $(n=195)$ participated in a survey study on health beliefs related to CVD, salt intake, physical activity (PA), and smoking in the fall of 2016.

Results: A high-salt diet, physical inactivity, and smoking were major lifestyle problems. Participants who adhered to a low-salt diet considered themselves to be susceptible to CVD. Most participants did not engage in regular PA. Regular PA was associated with less perceived susceptibility to CVD and greater perceived benefits of a healthy lifestyle for decreasing the likelihood of CVD.

Conclusions: Each refugee population may require individualized strategies to promote PA and a healthy diet. Future studies should develop health education programs that are specifically designed for Karen refugees and evaluate such programs. In addition to health education programs on healthy lifestyle choices, tobacco cessation programs seem to be necessary for Karen refugees. At the same time, it is important to foster strategies to increase the utilization of preventive care among this population by promoting free or reduced-fee resources in the community to further promote their health.

Key words: Cardiovascular disease, Health beliefs, Diet, Physical activity, Karen refugees, Refugee resettlement

\section{INTRODUCTION}

The Karen are one of the refugee populations resettled in the US. Civil war followed by the independence of Myanmar,

Received: July 1, 2017 Accepted: November 2, 2017

Corresponding author: Akiko Kamimura, PhD

380 S 1530 E, Salt Lake City, UT 84112, USA

Tel: +1-801-585-5496, Fax: +1-801-585-3784

E-mail: akiko.kamimura@utah.edu

This is an Open Access article distributed under the terms of the Creative Commons Attribution Non-Commercial License (http://creativecommons.org/licenses/by$\mathrm{nc} / 4.0 /$ ) which permits unrestricted non-commercial use, distribution, and reproduction in any medium, provided the original work is properly cited. also known as Burma, in 1948 caused the conflict that has displaced a number of Karen ethnic tribes to refugee camps along the Thai-Myanmar border [1]. Of the approximately 150000 refugees at the Thai-Myanmar border, over 60000 have resettled in a third country, most commonly the US [2]. Previous studies on the health of Karen refugees in the US have mainly focused on mental health [3,4]. Since nearly $90 \%$ of Karen refugees resettled in the US have experienced war trauma, forms of psychological distress such as depression, post-traumatic disorders, and somatic symptoms are significant mental health issues among this population [4]. Due to the continued violence in Myanmar, Karen refugees will reside 
in the US for long term or permanently [1]. As Karen people live in the US longer, research into their health and corresponding practical guidelines are increasingly needed, not only for mental health issues, but also for other chronic conditions such as cardiovascular disease (CVD). For example, a study conducted in Denmark suggested that the risk of stroke among refugees increased as the duration of their residence in a resettled country became longer [5]. However, little is known about CVD-related issues among Karen refugees in the US.

In general, refugees resettled in the US may be at risk for CVD [6]. For example, the prevalence of diabetes, hypertension, and hyperlipidemia has been found to be higher among Cambodian refugees than the US general population [6]. Somali immigrants and refugees reported high CVD risk factors (e.g., a prevalence of diabetes of $12.1 \%$ among Somali patients, vs. 5.3\% in non-Somali patients) [7]. However, the current health services for refugees lack long-term follow-up for chronic health conditions [8]. Lifestyle issues, such as physical activity (PA), diet, and smoking, are important for preventing and/or managing CVD [9]. In general, immigrants who migrate from an agricultural, rural, or small-town setting to an industrialized town experience reduced PA and increased intake of high-fat food and salt [10]. In addition, underserved immigrant populations encounter financial and cultural barriers to PA [11]. Thus, physical inactivity is a common issue among refugees. The lack of familiarity and comfort with starting PA in a new country are common barriers preventing Cambodian, Mexican, Somali, and Sudanese immigrants and refugees from starting an active lifestyle [12]. Bhutanese refugees consume higher quantities of vegetables than the US general population, but tend to be physically inactive [13]. Moreover, underserved immigrant populations may consume foods from their ethnic backgrounds in the community, if available, but are also exposed to the convenience of fast food and other dietary changes that can bring negative impacts on their health [14]. Furthermore, some studies reported a high prevalence of tobacco smoking among refugees. For example, a higher prevalence of tobacco smoking (16.7\%) compared to the general public (14.4\%) was found among refugees from Iran, Ukraine, and Vietnam in California [15]. Approximately half of Somali refugees in Minnesota reported tobacco use [16]. However, knowledge about CVD-related health beliefs and lifestyle issues among Karen refugees resettled in the US is lacking. To develop effective CVD prevention programs for Karen refugees, such knowledge is important.
The purpose of this study was to examine CVD-related health beliefs and lifestyle issues (PA, diet, and smoking) among Karen refugees resettled in the US from the ThaiMyanmar border. Because Karen refugees in the US are relatively young, CVD may not be a current health problem. However, it may become a problem in the future. The conceptual framework of this study is the Health Belief Model (HBM). The HBM is a useful framework to describe health behaviors for health behavior interventions and includes perceived susceptibility, severity, benefits, and barriers as constructs that affect individual health behaviors [17].

\section{METHODS}

\section{Data Collection and Participants}

This study was approved by the institutional review board. Data were collected using a self-administered or intervieweradministered survey. Two student research assistants who had themselves migrated to the US from a refugee camp at the Thai-Myanmar border were fluent in the languages spoken by Karen refugees. Participants were recruited through in-person contacts, flyers, snowball sampling, and professional and personal networks in the refugee community and community organizations that serve refugees. The survey instrument and consent cover letter were available in English. Participants who were fluent in English took the survey as self-administered. Participants who were not fluent in English took the survey as interviewer-administered. Consent was obtained from each participant. No personal information was gathered from the participants. Participants received a small gift (around US dollar 1 in value - e.g., a bandana, a hat, or a pair of gloves) after completing the survey. Approximately 1000 Karen refugees live in Salt Lake County, UT, where this study was conducted.

\section{Measures}

\section{Health beliefs related to cardiovascular disease}

Health beliefs related to CVD were measured using the Health Beliefs Related to Cardiovascular Disease (HBCVD) instrument, which has a 4-point Likert scale (from 1 [strongly disagree] to 4 [strongly agree]) [18]. The HBCVD scale includes 25 items with 4 subscales, including perceived susceptibility to CVD ( 5 items), perceived severity of CVD ( 5 items), the benefits of having a healthy diet and engaging in exercise (6 items), and barriers to healthy diet and exercise (9 items). Be- 
low are examples for each subscale: "It is likely I will suffer from a heart attack or stroke in the future" (susceptibility), "Having a heart attack or stroke is always fatal” (severity), "When I exercise I am doing something good for myself" (benefits, with reverse coding), and "It is painful for me to walk for more than 5 minutes" (barriers). Scoring was based on the mean of the items in the same subscale. Higher scores indicated higher levels of susceptibility, severity, or barriers, or lower levels of benefits. The Cronbach alpha values for this study population were: 0.909 (susceptibility), 0.790 (severity), 0.930 (benefits), and 0.718 (barriers). While the authors who developed this scale suggested that the barriers and severity subscales had poor internal consistency [18], the reliability of all subscales for this study population was acceptable.

\section{Salt intake, physical activity, and smoking}

Salt intake, PA, and smoking were assessed using the Hypertension Self-Care Activity Level Effects (H-SCALE) instrument [19]. The H-SCALE asks the number of days in the last week that a participant engaged in each item. For salt intake, the following 2 items were included: "Salt your food at the table" and "Add salt to food when you're cooking." For PA, there were 2 items: "Do at least 30 minutes total of PA" and "Do a specific exercise activity (such as swimming, walking, or biking) other than what you do around the house or as part of your work." One item, "Smoke a cigarette or cigar, even just one puff," asked about tobacco smoking. For salt intake, if the sum of the 2 items was 6 or lower, the participant was considered to have a low-salt diet. Regular PA was defined by having a combined score of 8 or higher for the 2 items. For tobacco smoking, participants who answered 1 day or more were considered smokers.

\section{Perceived benefits of and barriers to physical activity}

A measure of perceived benefits of and barriers to PAs was developed based on the document from the National Institute of Health entitled "Changing your habits: steps to better health" [20]. The instrument included 5 benefit items and 5 barrier items. Participants were asked how each item would fit their perceptions about the impact of PAs on their feelings or lifestyle using a 5-point Likert scale (from $1=$ [strongly disagree] to $5=$ [strongly agree]). The benefit sub-scale included, "improve my health," "reduce my risk for serious health problems," "feel better about myself," "become stronger," and "have fun." The barrier sub-scale included "do not have time," "do not have the energy," "do not have enough money," "am not good at being active," and "do not know what to do." Scoring was based on the mean of all points collected. Higher scores indicated higher levels of benefits or barriers. The Cronbach alpha values for the sub-scales were 0.945 for benefits and 0.951 for barriers.

\section{Socio-demographic characteristics}

The survey asked for the following socio-demographic characteristics: age, sex, educational attainment, marital status, house ownership, automobile ownership, employment status, years residing in the US, and religion. In addition, participants were asked healthcare-related questions about health insurance, dental insurance, physical exam in the past year, the utilization of an emergency room in the past year, the utilization of a free clinic, preference for a female physician, preference for a male physician, and preference for a physician from the same cultural background.

\section{Data Analysis}

Data were analyzed using SPSS version 22 (IBM Corp., Armonk, NY, USA). Descriptive statistics were used to describe the characteristics of the participants and variables. Multiple linear regression was performed to examine the association between CVD-related health beliefs and demographic characteristics, lifestyle (low-salt diet, regular PA, and smoking), and benefits of and barriers to PA. The skewness of the variables and collinearity were tested prior to the regression analysis. There was no significant skewness in the distribution or collinearity.

\section{RESULTS}

Table 1 presents the characteristics of the participants $(n=195)$ and descriptive statistics. All participants were refugees from the Thai-Myanmar border. Nearly half of the participants were females (46.7\%). The average age of the participants was 35.91 years (standard deviation [SD], 15.84 years). On average, the participants had lived in the US for 6.67 years (SD, 2.76 years). Slightly over one-third of the participants had completed high school or a higher level of formal education (35.9\%). Approximately half of the participants were married (48.7\%). Nearly $60 \%$ of the participants had a full-time job (57.9\%). More than one-third of the participants or their family owned a house (37.4\%). Nearly $80 \%$ of the participants or their family owned a car (77.9\%). Half of the participants self- 
Table 1. Socio-demographic characteristics of participants and descriptive statistics $(n=195)$

\begin{tabular}{lc}
\hline Characteristics & $\begin{array}{c}\text { Descriptive } \\
\text { statistics }\end{array}$ \\
\hline Female & $91(46.7)$ \\
High school or higher & $70(35.9)$ \\
Married & $95(48.7)$ \\
Work full-time & $113(57.9)$ \\
House owner (or owner's family) & $73(37.4)$ \\
Car owner (or owner's family) & $152(77.9)$ \\
Religion & \\
Christian & $98(50.3)$ \\
Buddhist & $70(35.9)$ \\
No health insurance & $60(30.8)$ \\
No dental insurance & $86(44.1)$ \\
Had physical exam in the past year & $30(15.4)$ \\
Used emergency room in the past year & $11(5.6)$ \\
Had used a free clinic & $26(13.3)$ \\
Prefer a female physician & $35(17.9)$ \\
Prefer a male physician & $8(4.1)$ \\
Prefer a physician from the same cultural background & $48(24.6)$ \\
Low-salt diet & $50(25.6)$ \\
Regular PA & $36(18.5)$ \\
Smoker & $37(19.0)$ \\
Age & $3.52 \pm 0.56$ \\
Years in the US & $3.50 \pm 0.89 \pm 0.969 .97 \pm 2.84$ \\
\hline Health beliefs related to CVD & \\
Perceived susceptibility & $2.39 \pm 1.00$ \\
Perceived severity & $2.62 \pm 0.88$ \\
Benefits of having a healthy diet and engaging in & $2.11 \pm 0.89$ \\
\hline
\end{tabular}

Values are presented as frequency (\%) or mean \pm standard deviation.

PA, physical activity; CVD, cardiovascular disease.

${ }^{1}$ Higher scores indicate higher levels of susceptibility, severity or barriers, or lower levels of benefits.

${ }^{2}$ Higher scores indicate higher levels of benefits or barriers.

identified as Christians (50.3\%), followed by Buddhists (35.9\%). The remaining participants reported that they were Muslim or did not have any religious belief (data not shown).

While over $40 \%$ of the participants did not have dental insurance $(44.1 \%)$, fewer than one-third of the participants did not have health insurance (30.8\%). Only $15.4 \%$ of the participants reported having a physical exam in the past year. The utilization of an emergency room or free clinic in the past year was low (5.6 and 13.3\%, respectively). The sex preference for
Table 2. Predictors' ${ }^{1}$ of health beliefs related to cardiovascular disease

\begin{tabular}{lcc}
\hline & Susceptibility $\boldsymbol{B}^{\mathbf{2}}$ & Benefits $\boldsymbol{B}^{\mathbf{3}}$ \\
\hline Age & $<0.01$ & -0.01 \\
Female & -0.05 & 0.17 \\
High school or higher & -0.04 & $-0.49^{*}$ \\
Married & 0.08 & 0.04 \\
Full time employment & 0.25 & -0.06 \\
Years in the US & -0.04 & 0.04 \\
No health insurance & 0.27 & -0.05 \\
Low-salt diet & $0.45^{* *}$ & -0.17 \\
Regular PA & 0.30 & -0.23 \\
Smoker & 0.36 & 0.19 \\
Benefits of PA & $-0.21^{*}$ \\
Barriers to PA & $42^{* *}$ & 0.14 \\
(Constant) & 0.13 & $2.52^{* *}$ \\
$R^{2}$ & $2.71^{* *}$ & 0.13 \\
$F$ & 0.23 & 1.92 \\
$p$-value & 4.01 & $<0.05$ \\
\hline
\end{tabular}

PA, physical activity.

'Multivariate multiple regression.

${ }^{2}$ Higher scores indicate higher levels of perceived susceptibility.

${ }^{3}$ Higher scores indicate lower levels of perceived benefits related to cardiovascular disease.

${ }^{4}$ Higher scores indicate higher levels of benefits or barriers.

${ }^{*} p<0.05,{ }^{* *} p<0.01$.

physicians was $17.9 \%$ for a female physician and $4.1 \%$ for a male physician. Additionally, one-third of the female participants preferred a female physician (data not shown). Approximately 1 in 4 of the participants expressed a preference for a physician from the same cultural background (24.6\%). Onequarter of the participants reported consuming a low-salt diet (25.6\%). Fewer than $20 \%$ of the participants reported regular PA (18.5\%). The most common PA was walking (56.4\% of all participants). Nineteen percent of the participants were current smokers. The prevalence of smoking was higher among females (22.0\%) than males (16.3\%) (data not shown).

Table 2 presents the results of the regression analysis. Analysis of variance was not significant for severity or barriers, so only 2 models that had severity or benefits as a dependent variable were included. A low-salt diet and greater perceived benefits of PA on their feelings/lifestyle were associated with higher levels of perceived susceptibility to CVD $(p<0.01)$. Having attained a high school diploma or higher and greater perceived benefits of PA on their feelings/lifestyle were associated with greater perceived benefits of a healthy lifestyle for decreasing the likelihood of CVD. 


\section{DISCUSSION}

This study examined CVD-related health beliefs and lifestyle issues (PA, diet, and smoking) among Karen refugees resettled in the US from the Thai-Myanmar border. The results of the descriptive statistics of this study (Table 1) indicate that a highsalt diet, physical inactivity, and smoking were major lifestyle problems that could affect the likelihood of CVD among Karen refugees. Despite poor health behaviors, there was a high percentage of Karen refugees who were uninsured, but had not used available free healthcare resources such as free clinics. This study has 3 main findings. First, while high-salt intake was a common dietary problem, those who adhered to a low-salt diet perceived themselves to be susceptible to CVD. Second, most Karen refugees did not engage in regular PA. Taking part in regular PA was associated with (1) lower levels of perceived susceptibility to CVD and (2) greater perceived benefits of a healthy lifestyle for reducing the chance of getting CVD. Third, the prevalence of tobacco smoking seemed to be high among Karen refugees.

While dietary recommendations to prevent CVD include a high intake of fresh vegetables and fruits, low saturated fat and cholesterol, high fiber, and low-salt/sodium [9], promoting a low-salt/sodium diet seems to be a priority for promoting a healthy diet among Karen refugees. Because this study did not ask about salt intake before migration, we could not obtain any information about possible changes in salt intake. However, high-salt intake is a global public health issue, and is particularly problematic in some Asian countries [21]. It is possible that Karen refugees had a high-salt intake before their migration to the US. Since the results of this study indicate that consuming a low-salt diet was associated with greater perceived susceptibility to CVD, salt intake interventions can promote changes in diet while increasing awareness of CVD. However, it is very challenging to change one's eating habits to reduce salt intake [22]. Health promotion programs to reduce salt intake in a way that fits the diet of Karen refugees need to be developed.

Only a small percentage of the participants engaged in regular PA. This suggests that PA should be promoted among Karen refugees. The percentage observed in this study is lower than what has been reported in other refugee populations, such as Bhutanese refugees (41\%) [14]. Underserved populations often experience barriers to PA, such as lack of time, cost, lack of childcare, and low awareness of the importance of PA [23]. It is possible that Karen refugees have additional barriers to PA. Among Cambodian and Iraqi refugees in the US, higher levels of acculturation were associated with higher levels of PA $[24,25]$. To promote PA among Karen refugees, it may be effective for health education programs to include acculturation interventions.

The prevalence of current tobacco smoking among the participants of this study was higher than that of the US general population (15.1\% in 2015) [26]. Although tobacco use did not have any impact of CVD-related health beliefs, our results suggest a possible lack of awareness of the association between tobacco smoking and CVD. Developing tobacco cessation programs for Karen refugees is necessary to promote their health. A study conducted in Australia reported a high prevalence of tobacco smoking among male Burmese refugees and recommended smoking cessation interventions that consider betel nut intake [27]. Among Southeast Asian American youths, tobacco smoking is often associated with other kinds of substance use [28]. Future research should develop, implement, and evaluate tobacco cessation programs for Karen refugees to prevent the health issues that arise from tobacco smoking, as well as other forms of habitual substance use.

While this study provides insightful information about CVDrelated health beliefs and lifestyle issues among Karen refugees, who have not been well studied, it has some limitations. This study had a cross-sectional design, and did not necessarily examine causal directions among the variables. The participants were a convenience sample and may not have been representative of the Karen refugees in the US. It is possible that relatively educated individuals were recruited. The measures of perceived benefits and barriers did not include smoking, which is a major behavioral factor. The sample size $(n=195)$ was small. However, given the number of Karen refugees in Salt Lake County (approximately 1000), the sample size can be considered reasonable. While the target population was approximately 1000 in this county, more than 50000 Karen refugees have resettled in over 40 states in the US [29]. The results of this study may suggest implications regarding Karen refugees resettled in other counties or states. Furthermore, since we did not have a comparison group, this study alone cannot be interpreted as reflecting unique factors of Karen refugees related to their perceived CVD risk. Further studies that include comparison groups would be necessary to identify unique aspects of Karen refugees related to their perceptions of CVD risk. 
This study provides important information that would be useful for developing CVD prevention and management strategies for Karen refugees resettled in the US or other developed countries. A study on Hispanic, Somali, and Sudanese immigrants/refugees showed that a community-based PA and nutrition intervention was effective for reducing CVD risk [30]. However, the results of the present study indicate that each refugee population may require individualized strategies to promote PA and a healthy diet. Future studies should develop and evaluate health education programs that are specifically designed for Karen refugees. In addition to health education programs on healthy lifestyles, tobacco cessation programs seem to be necessary for Karen refugees. At the same time, it is important to foster strategies to increase the utilization of preventive care among this population by promoting free or reduced-fee resources in the community to further promote their health.

\section{ACKNOWLEDGEMENTS}

This study was supported by the College of Social and Behavioral Science (Akiko Kamimura) and the Undergraduate Research Opportunities Program (Kai Sin and Mu Pye), University of Utah. We thank Maziar Nourian for his input on this study.

\section{CONFLICT OF INTEREST}

The authors have no conflicts of interest associated with the material presented in this paper.

\section{ORCID}

Akiko Kamimura https://orcid.org/0000-0001-6528-5770

\section{REFERENCES}

1. Kenny P, Lockwood-Kenny K. A mixed blessing: Karen resettlement to the United States. J Refug Stud 2011;24(2):217-238.

2. US Department of State. U.S. relations with Burma [cited 2017 Feb 20]. Available from: https://www.state.gov/r/pa/ei/bgn/ 35910.htm.

3. Cook TL, Shannon PJ, Vinson GA, Letts JP, Dwee E5. War trauma and torture experiences reported during public health screening of newly resettled Karen refugees: a qualitative study. BMC Int Health Hum Rights 2015;15:8.

4. Shannon PJ, Vinson GA, Wieling E, Cook T, Letts J. Torture, war trauma, and mental health symptoms of newly arrived Karen refugees. J Loss Trauma 2015;20(6):577-590.

5. Norredam M, Agyemang C, Hoejbjerg Hansen OK, Petersen $\mathrm{JH}$, Byberg S, Krasnik A, et al. Duration of residence and disease occurrence among refugees and family reunited immigrants: test of the 'healthy migrant effect' hypothesis. Trop Med Int Health 2014;19(8):958-967.

6. Marshall GN, Schell TL, Wong EC, Berthold SM, Hambarsoomian $\mathrm{K}$, Elliott MN, et al. Diabetes and cardiovascular disease risk in Cambodian refugees. J Immigr Minor Health 2016;18(1): 110-117.

7. Njeru JW, Tan EM, St Sauver J, Jacobson DJ, Agunwamba AA, Wilson PM, et al. High rates of diabetes mellitus, pre-diabetes and obesity among Somali immigrants and refugees in Minnesota: a retrospective chart review. J Immigr Minor Health 2016;18(6):1343-1349.

8. Pace M, Al-Obaydi S, Nourian MM, Kamimura A. Health services for refugees in the United States: policies and recommendations. Public Policy Adm Res 2015;5(8):63-68.

9. Centers for Disease Control and Prevention. Prevention: what you can do [cited 2017 Feb 20]. Available from: https://www. cdc.gov/heartdisease/what_you_can_do.htm.

10. Rosenthal T. The effect of migration on hypertension and other cardiovascular risk factors: a review. J Am Soc Hypertens 2014;8(3):171-191.

11. Kamimura A, Ashby J, Trinh H, Prudencio L, Mills A, Tabler J, et al. Uninsured free clinic patients' experiences and perceptions of healthcare services, community resources, and the Patient Protection and Affordable Care Act. Patient Exp J 2016;3(2): 12-21.

12. Wieland ML, Tiedje K, Meiers SJ, Mohamed AA, Formea CM, Ridgeway $\mathrm{JL}$, et al. Perspectives on physical activity among immigrants and refugees to a small urban community in Minnesota. J Immigr Minor Health 2015;17(1):263-275.

13. Misra SM, Nepal VP, Banerjee D, Giardino AP. Chronic health conditions, physical activity and dietary behaviors of Bhutanese refugees: a Houston-based needs assessment. J Immigr Minor Health 2016;18(6):1423-1431.

14. Tiedje K, Wieland ML, Meiers SJ, Mohamed AA, Formea CM, Ridgeway JL, et al. A focus group study of healthy eating knowledge, practices, and barriers among adult and adolescent immigrants and refugees in the United States. Int J Behav Nutr Phys Act 2014;11:63. 
15. Nguyen MT, Rehkopf DH. Prevalence of chronic disease and their risk factors among Iranian, Ukrainian, Vietnamese refugees in California, 2002-2011. J Immigr Minor Health 2016;18 (6):1274-1283.

16. Giuliani KK, Mire OA, Jama S, Dubois DK, Pryce D, Fahia S, et al. Tobacco use and cessation among Somalis in Minnesota. Am J Prev Med 2008;35(6 Suppl):S457-S462.

17. Champion VL, Skinner CS. The health belief model. In: Glanz K, Rimer BK, Viswanath K, editors. Health behavior and health education: theory, research, and practice. 4th ed. San Francisco: Jossey-Bass; 2008, p. 45-96.

18. Tovar EG, Rayens MK, Clark M, Nguyen H. Development and psychometric testing of the Health Beliefs Related to Cardiovascular Disease Scale: preliminary findings. J Adv Nurs 2010; 66(12):2772-2784.

19. Warren-Findlow J, Seymour RB. Prevalence rates of hypertension self-care activities among African Americans. J Natl Med Assoc 2011;103(6):503-512.

20. National Institute of Diabetes and Digestive and Kidney Diseases. Changing your habits for better health [cited 2017 Feb 20]. Available from: https://www.niddk.nih.gov/health-information/diet-nutrition/changing-habits-better-health.

21. Brown IJ, Tzoulaki I, Candeias V, Elliott P. Salt intakes around the world: implications for public health. Int J Epidemiol 2009;38(3):791-813.

22. Aung MN, Yuasa M, Moolphate $S$, Nedsuwan S, Yokokawa $H$, Kitajima T, et al. Reducing salt intake for prevention of cardiovascular diseases in high-risk patients by advanced health education intervention (RESIP-CVD study), Northern Thailand: study protocol for a cluster randomized trial. Trials 2012;13: 158.

23. Withall J, Jago R, Fox KR. Why some do but most don't. Barri- ers and enablers to engaging low-income groups in physical activity programmes: a mixed methods study. BMC Public Health 2011;11:507.

24. Jen KL, Zhou K, Arnetz B, Jamil H. Pre- and post-displacement stressors and body weight development in Iraqi refugees in Michigan. J Immigr Minor Health 2015;17(5):1468-1475.

25. Nelson-Peterman JL, Toof R, Liang SL, Grigg-Saito DC. Longterm refugee health: health behaviors and outcomes of Cambodian refugee and immigrant women. Health Educ Behav 2015;42(6):814-823.

26. Centers for Disease Control and Prevention. Current cigarette smoking among adults in the United States [cited 2017 Feb 20]. Available from: https://www.cdc.gov/tobacco/data_statistics/fact_sheets/adult_data/cig_smoking/.

27. Furber S, Jackson J, Johnson K, Sukara R, Franco L. A qualitative study on tobacco smoking and betel quid use among Burmese refugees in Australia. J Immigr Minor Health 2013;15 (6):1133-1136.

28. Lee JP, Battle RS, Lipton R, Soller B. 'Smoking': use of cigarettes, cigars and blunts among Southeast Asian American youth and young adults. Health Educ Res 2010;25(1):83-96.

29. Burmese American Community Institute. Burmese refugee population in the US [cited 2017 Oct 10]. Available from: https://web.archive.org/web/20170329163146/http://www. baci-indy.org/resources/burmese-refugee-population-in-theus\#_edn9.

30. Wieland ML, Weis JA, Hanza MM, Meiers SJ, Patten CA, Clark $M M$, et al. Healthy immigrant families: participatory development and baseline characteristics of a community-based physical activity and nutrition intervention. Contemp Clin Trials 2016;47:22-31 